\title{
Neonatal Outcomes After Elective Cesarean Delivery
}

\author{
Beena D. Kamath, MD, MPH, James K. Todd, MD, Judith E. Glazner, MS, Dennis Lezotte, PhD, \\ and Anne M. Lynch, MD, MSPH
}

OBJECTIVE: To examine the outcomes of neonates born by elective repeat cesarean delivery compared with vaginal birth after cesarean (VBAC) in women with one prior cesarean delivery and to evaluate the cost differences between elective repeat cesarean and VBAC.

METHODS: We conducted a retrospective cohort study of 672 women with one prior cesarean delivery and a singleton pregnancy at or after 37 weeks of gestation. Women were grouped according to their intention to have an elective repeat cesarean or a VBAC (successful or failed). The primary outcome was neonatal intensive care unit (NICU) admission and measures of respiratory morbidity.

RESULTS: Neonates born by cesarean delivery had higher NICU admission rates compared with the VBAC group $(9.3 \%$ compared with $4.9 \%, P=.025)$ and higher rates of oxygen supplementation for delivery room resuscitation $(41.5 \%$ compared with $23.2 \%, P<.01)$ and after NICU admission (5.8\% compared with $2.4 \%$, $P<.028)$. Neonates born by VBAC required the least delivery room resuscitation with oxygen, whereas neonates delivered after failed VBAC required the greatest degree of delivery room resuscitation. The costs of elective repeat cesarean were significantly greater than VBAC. However, failed VBAC accounted for the most expensive total birth experience (delivery and NICU use).

CONCLUSION: In comparison with vaginal birth after cesarean, neonates born after elective repeat cesarean

From the Departments of Pediatrics, Section of Neonatology, and Obstetrics and Gynecology, University of Colorado School of Medicine; Department of Infectious Disease and Epidemiology, the Children's Hospital, Denver; and Colorado School of Public Health, Denver, Colorado.

The authors thank Jocelyn Seelye, BS, Jan Hart, MPH, and Sarah Crowley, BA, for being the Research Assistants for the Perinatal Database.

Corresponding author: Beena D. Kamath, MD, MPH, Mail Stop 8402, Education 2 South, Room 4304, 13121 East 17th Avenue, PO Box 6508, Aurora, CO 80045; e-mail: Beena.Kamath@ucdenver.edu.

Financial Disclosure

The authors did not report any potential conflicts of interest.

(C) 2009 by The American College of Obstetricians and Gynecologists. Published by Lippincott Williams \& Wilkins.

ISSN: 0029-7844/09 delivery have significantly higher rates of respiratory morbidity and NICU-admission and longer length of hospital stay.

(Obstet Gynecol 2009;113:1231-8)

LEVEL OF EVIDENCE: II

In 2006, the United States cesarean delivery rate of $31.1 \%$ was at an all-time high, making cesarean delivery the most common surgical procedure performed in American women., ${ }^{1,2}$ This high rate of cesarean delivery is attributed to the rise in primary cesarean delivery rates from $14.6 \%$ in 1996 to $20.3 \%$ in 2005, an increase of $60 \% 0^{2,3}$ With the rates of vaginal births after cesarean delivery (VBAC) at an all-time low of $7.9 \%$ in 2005, women who have a primary cesarean delivery have a greater than $90 \%$ chance of having a repeat cesarean delivery, only serving to increase the overall cesarean delivery rate. ${ }^{2}$ Almost one half of cesarean deliveries, a rate of $15 \%$, are done electively, before the onset of labor.

Controversy remains on whether a trial of labor or an elective repeat cesarean delivery is preferable for a woman with a history of cesarean delivery. Historically, concerns regarding the increased risk of uterine rupture and perinatal asphyxia in trial of labor after cesarean compared with planned repeat cesarean have swayed obstetricians away from recommending a trial of labor after cesarean delivery; however, the absolute risk of perinatal asphyxia remains small. ${ }^{4,5}$ By far, the most frequent complication for the newborn after cesarean delivery is respiratory morbidity; therefore, to avoid iatrogenic prematurity, most obstetricians will not time the elective delivery of an neonate before 39 weeks of gestation without documentation of amniocentesis indicating fetal lung maturity. ${ }^{6}$

Furthermore, a paucity of data exists regarding the cost of elective repeat cesarean delivery as compared with other delivery options. Prior studies have 
lacked large enough patient numbers to confidently estimate the costs of the rare complications from each type of delivery, therefore demonstrating a clear need for further investigation into the costs of elective cesarean delivery. ${ }^{7,8}$

The aims of this study were to examine select neonatal outcomes of neonates born by elective repeat cesarean delivery and VBAC in women presenting for a subsequent delivery after one previous cesarean delivery, and to describe the cost differences between elective repeat cesarean delivery and VBAC delivery. We hypothesized that neonates born by elective repeat cesarean delivery would have greater respiratory morbidity resulting in admission to the NICU, which would propel hospital costs to increase.

\section{PATIENTS AND METHODS}

We performed a retrospective cohort study using records from the Perinatal Database of the Department of Obstetrics and Gynecology at the University of Colorado Denver for the period between October 1,2005 , and July 1,2008 . The study was approved by the Colorado Multiple Institutional Review Board. During the study period, 8,211 deliveries were recorded in the database. From this data set, we identified women of any parity with a history of one prior cesarean delivery who were pregnant with a singleton 37 weeks or more gestation without congenital anomalies $(\mathrm{n}=672)$. In this cohort, $343(51.0 \%)$ planned an elective repeat cesarean delivery, and were considered the "intended cesarean group," and 329 (49.0\%) planned a trial of labor, and were called the "intended VBAC group." The intended cesarean delivery group consisted of women who had a planned elective repeat cesarean delivery without labor (group 1) or a planned elective repeat cesarean delivery after onset of labor (group 2). The intended VBAC group included women who had successful vaginal birth after cesarean delivery (group 3), and a failed VBAC requiring emergent cesarean delivery (group 4). Medical record review was conducted to confirm correct classification of the category of delivery. Labor was defined as subjectively painful contractions more frequent than every 10 minutes, with the obstetrician's documentation of cervical change or the words "active labor" in the chart.

The primary outcome was admission to the NICU, defined as any length of time spent in the NICU after delivery, and included neonates who transitioned for a period of time due to a clinical assessment of respiratory distress. Maternal demographic characteristics analyzed as possible confounders were mother's age, parity, race or ethnicity (non-
Hispanic white, Hispanic, African American, and other), and maternal education (high school level or less, university level or more). Maternal medical characteristics included body mass index (BMI) calculated using prepregnancy weight and height, history of a successful VBAC, chronic medical disease (autoimmune disease, chronic hypertension, or preexisting diabetes), and diabetes during pregnancy (both preexisting and gestational diabetes). Pregnancy characteristics included evidence of macrosomia (neonate more than 90\% weight for gestational age), attempt at amniocentesis for fetal lung maturity, induction of labor, nonreassuring fetal heart tones, and finally, chorioamnionitis (documented by the obstetrician and defined as maternal fever plus one of the following: fundal tenderness, purulent discharge, and maternal tachycardia). Characteristics regarding the neonate included gestational age (based on last menstrual period or ultrasonography), sex, and birth weight. Neonatal outcomes included any oxygen use during delivery room resuscitation, highest level of delivery room resuscitation required (routine resuscitation only, blow-by oxygen, mask continuous positive airway pressure, bag or mask ventilation, or endotracheal intubation), neonate disposition (well baby nursery compared with NICU), endotracheal intubation outside the delivery room, hypoglycemia requiring NICU stay, respiratory distress requiring NICU stay (need for oxygen or other ventilatory support), and type of ventilatory support needed in NICU (conventional mechanical ventilation, oxygen hood, nasal cannula, or continuous positive airway pressure).

We obtained facility and physician charges for both mothers and their neonates by searching the medical invoices in the patient medical account for the hospital days during which the delivery occurred. We were unable to get charges for delivery complications that required readmission to the hospital. Facility charges included those for any supplies or facility employees (including nursing staff) needed to care for the patient, and physician charges included the time and services of the medical provider.

Because charges do not necessarily reflect the actual cost of resources used to provide hospital services and are often an artifact of accounting methods or of cross-subsidization among various hospital services, we used costs as our measure of resource use in this study. Costs were calculated using the University of Colorado hospital-wide cost-to-charge ratio of $27.79 \%$ for the births occurring before June 30, 2006, and $28.14 \%$ for births occurring afterward. These hospital-wide cost-to-charge ratios were lower than 
Table 1. Description of Maternal Risk Factors and Labor and Delivery Events Among Women of the Four Delivery Categories

\begin{tabular}{|c|c|c|c|c|c|}
\hline Maternal Risk Factors & $\begin{array}{c}\text { Elective Cesarean No } \\
\text { Labor }(n=239)\end{array}$ & $\begin{array}{l}\text { Elective Cesarean } \\
\text { Labor }(n=104)\end{array}$ & $\begin{array}{c}\text { VBAC } \\
(n=244)\end{array}$ & $\begin{array}{l}\text { Failed VBAC } \\
\quad(n=85)\end{array}$ & $P^{*}$ \\
\hline Maternal age (y) & $30.0(21,40)$ & $28.0(19,38)$ & $28.0(20,38)$ & $29.0(22,38)$ & .01 \\
\hline Parity & $1.0(1,4)$ & $1.0(1,3)$ & $2.0(1,4)$ & $1.0(1,5)$ & $<.001$ \\
\hline \multicolumn{6}{|l|}{ Race/ethnicity of mother } \\
\hline White, non-Hispanic & $90(38.1)$ & $30(29.7)$ & $74(30.4)$ & $21(24.7)$ & .31 \\
\hline Hispanic & $104(43.5)$ & $58(55.8)$ & $131(53.9)$ & $48(56.5)$ & \\
\hline African American & $30(12.6)$ & $9(8.7)$ & $22(9.1)$ & $11(12.9)$ & \\
\hline Other & $15(6.3)$ & $7(6.7)$ & $16(6.6)$ & $5(5.9)$ & \\
\hline \multicolumn{6}{|l|}{ Maternal education } \\
\hline High school or less & $167(69.9)$ & $83(79.8)$ & $198(81.2)$ & $63(74.1)$ & .024 \\
\hline University/postgraduate & $72(30.1)$ & $21(20.2)$ & $46(18.9)$ & $22(26.5)$ & \\
\hline Body mass index & $26.4(19.3,41.4)$ & $25.7(19.3,37.7)$ & $24.4(19.0,37.8)$ & $25.0(19.4,40.4)$ & .032 \\
\hline Chronic medical disease $^{\dagger}$ & $14(5.9)$ & $1(1.0)$ & $4(1.6)$ & $5(5.9)$ & .022 \\
\hline Had prior successful VBAC & $16(6.7)$ & $6(5.8)$ & $87(35.7)$ & $10(11.8)$ & $<.001$ \\
\hline \multicolumn{6}{|l|}{ Pregnancy and labor } \\
\hline Amniocentesis for FLM & $22(9.2)$ & $7(6.7)$ & $2(0.8)$ & $1(1.2)$ & $<.001$ \\
\hline Induction of labor & $3(1.3)$ & $1(1.0)$ & $38(15.6)$ & $27(31.6)$ & $<.001$ \\
\hline NRFHTs & $13(5.4)$ & $11(10.6)$ & $31(12.8)$ & $21(24.7)$ & $<.001$ \\
\hline Chorioamnionitis & $4(1.7)$ & $4(3.9)$ & $10(4.1)$ & $14(16.5)$ & $<.001$ \\
\hline Meconium at delivery & $14(5.9)$ & $15(14.4)$ & $51(20.9)$ & $24(28.2)$ & $<.001$ \\
\hline
\end{tabular}

VBAC, vaginal birth after cesarean; FLM, fetal lung maturity; NRFHT, nonreassuring fetal heart tone.

Data are median $(5 \%, 95 \%)$ or $\mathrm{n}(\%)$.

* Continuous variables and categorical variables were compared by Kruskal Wallis and $\chi^{2}$ analyses, respectively.

$\uparrow$ Chronic medical disease includes autoimmune disease, hypertension, diabetes.

the actual cost-to-charge ratios for services on labor and delivery $(38.38 \%$ and $31.35 \%)$ but higher than those for the NICU $(23.72 \%$ and $20.92 \%)$ for the same time periods, respectively. Because we did not have more detailed lists of itemized charges, we used the hospital-wide cost-to-charge ratio.

To report all amounts in 2008 dollars, costs were adjusted yearly for inflation, and were also discounted at three percent annually. ${ }^{9}$ We adjusted for inflation using the yearly Consumer Price Index for the years 2005 to 2008 for medical care in the Denver-BoulderGreeley statistical area. ${ }^{10}$ The total amount for the birth, including delivery and NICU use fees, was calculated by summing the totals of the physician and facility costs for the mothers and their neonates.

Based on preliminary analysis with a sample size of 695 , we calculated a $90 \%$ power to detect a fourfold difference in NICU admission rates between the intended cesarean delivery group (10\%), and the intended VBAC group (2.5\%), based on a level of significance at $\alpha=0.05$.

The data were analyzed using SAS 9.2 (SAS Institute Inc., Cary, NC). Differences between categorical and continuous variables were tested using the $\chi^{2}$ and Kruskal-Wallis tests, respectively. Multivariable logistic regression was used to estimate the odds ratio of NICU admission for the three study groups compared with successful VBAC, adjusting for the covariates selected based on significant differences seen in univariable analysis or after consideration from past clinical evidence.

\section{RESULTS}

The overall cesarean delivery rate for the University of Colorado Hospital during the time period studied was $24.6 \%$, with a primary cesarean delivery rate of $15.5 \%$. Table 1 shows the general characteristics of the four study groups. Of the 672 women included in the study, $51 \%(\mathrm{n}=343)$ were in the intended elective repeat cesarean delivery, and $49 \%(\mathrm{n}=329)$ were the intended VBAC group. Of the total study population, $35.6 \%$ had a planned elective repeat cesarean delivery without labor $(\mathrm{n}=239), 15.5 \%$ had an elective repeat cesarean delivery after onset of labor $(\mathrm{n}=104), 36.3 \%$ had a successful VBAC $(\mathrm{n}=244)$, and $12.6 \%$ had a failed VBAC requiring emergent cesarean delivery $(\mathrm{n}=85)$. We found that older women and mothers with university or postgraduate education tended to have elective repeat cesarean delivery without labor. Women with successful VBAC deliveries had the greatest parity and the highest rates of prior VBAC compared with the other three groups. Significantly higher rates of chorioamnionitis and nonreassuring fetal heart tones were seen in the failed VBAC group than in the other three groups. More amniocenteses were done in women who underwent elective repeat 
Table 2. Comparison of Neonate Risk Factors and Neonatal Outcomes Among Women With an Intended Cesarean or Intended Vaginal Birth After Cesarean Delivery

\begin{tabular}{lccc}
\hline & $\begin{array}{c}\text { Intended } \\
\text { Cesarean } \\
(\mathbf{n}=343)\end{array}$ & $\begin{array}{c}\text { Intended } \\
\text { VBAC } \\
(\mathbf{n}=329)\end{array}$ & $\boldsymbol{P}^{*}$ \\
Neonate Characteristic & & & \\
Neonate disposition & $311(90.7)$ & $313(95.1)$ & .025 \\
Well baby nursery & $32(9.3)$ & $16(4.9)$ & \\
NICU & $39.0 \pm 0.97$ & $39.5 \pm 1.19$ & $<.001$ \\
Neonate gestational age (wk) & $142(41.5)$ & $76(23.2)$ & $<.001$ \\
Oxygen during delivery room & 14001 & & \\
$\quad$ resuscitation & & & \\
Delivery room resuscitation & & & \\
$\quad$ required & $200(58.3)$ & $251(76.3)$ & $<.001$ \\
Routine (drying and & & & \\
$\quad$ stimulation) & $100(29.2)$ & $43(13.1)$ & \\
Blow-by oxygen & $32(9.3)$ & $13(4.0)$ & \\
Mask CPAP & $8(2.3)$ & $11(3.3)$ & \\
Bag/mask ventilation & $2(0.6)$ & $8(2.4)$ & \\
Intubation & $12(3.5)$ & $3(0.9)$ & .03 \\
Admission for hypoglycemia & $20(5.8)$ & $8(2.4)$ & .028 \\
Oxygen requirement in NICU & $1(0.3)$ & $2(0.6)$ & \\
Conventional ventilation & $1(1.8)$ & $1(0.3)$ & \\
Oxyhood & $7(2.0)$ & $2(0.6)$ & \\
Cannula & $6(1.8)$ & $3(0.9)$ & \\
CPAP & & &
\end{tabular}

VBAC, vaginal birth after cesarean; NICU, neonatal intensive care unit; CPAP, continuous positive airway pressure.

Data are mean \pm standard deviation or $\mathrm{n}(\%)$.

* Continuous variables and categorical variables were compared by Kruskal Wallis and $\chi^{2}$ analyses, respectively. Fisher exact test was used in the case of a cell with $\mathrm{n}<5$.

cesarean delivery without labor $(9.2 \%)$, as compared with those women who had successful VBAC $(0.8 \%$, $P<.001)$. No differences were seen in regard to race or ethnicity, or rates of diabetes or macrosomia among the four delivery groups.

Table 2 documents the differences seen in the neonates born in the intended elective repeat cesarean delivery and intended VBAC groups. No differences were seen in the distribution of male neonates or birth weight between groups. For the primary outcome, admission to the NICU, the incidence was $7.1 \%(\mathrm{n}=48)$ in the full cohort of neonates, which included $9.3 \%$ of neonates born by intended elective repeat cesarean delivery and $4.9 \%$ of neonates born by intended VBAC $(P=.025)$. Our results show that significantly greater numbers of neonates in the intended cesarean group required blow-by oxygen and continuous positive airway pressure in the delivery room, ongoing oxygen supplementation once admitted to the NICU, and higher rates of admission for hypoglycemia. Notably, a larger number of neonates born in the intended VBAC group required bag mask ventilation $(3.3 \%)$ and endotracheal intubation $(2.4 \%)$ in the delivery room than neonates born in the intended cesarean group $(2.3 \%$ and $0.6 \%$, respectively, $P<.001$ ).

As shown in Table 3, rates of NICU admission were similar in elective repeat cesarean delivery with or without labor. Neonates born due to emergent cesarean delivery after failed VBAC had NICU admission rates comparable to the elective repeat cesarean delivery group, whereas neonates born after successful VBAC had the lowest rate of NICU admission. Neonates born in either intended cesarean delivery group experienced significantly higher rates of oxygen need and continuous positive airway pressure use in the delivery room than the successful VBAC group. However, neonates born by failed VBAC required the most significant measures of delivery room resuscitation, including bag or mask ventilation and endotracheal intubation, than did the other three groups. Neonates born by successful VBAC required the least amount of delivery room resuscitation, whereas neonates born by failed VBAC had rates of oxygen supplementation similar to neonates born by cesarean delivery.

The multivariable logistic regression analysis (Table 4) shows that after adjustment for other covariates (maternal education level, chronic medical disease, amniocentesis performed for fetal lung maturity, chorioamnionitis, nonreassuring fetal heart tones, and gestational age in weeks) and compared with neonates born by successful VBAC, neonates born by elective repeat cesarean delivery without labor continued to demonstrate significantly higher odds of admission to the NICU.

After the finding that the neonates born by successful VBAC had the least amount of respiratory morbidity requiring NICU admission and respiratory support, and the neonates born by failed VBAC required the greatest amount of resuscitation and respiratory support, we therefore performed a subanalysis to identify predictors of failed VBAC. After adjusting for maternal age, maternal race, BMI, parity, chorioamnionitis, nonreassuring fetal heart tones, induction of labor, and history of prior VBAC, significant covariates for failed VBAC were chorioamnionitis (adjusted odds ratio [OR] 5.58, 95\% confidence interval [CI] 2.08-14.99), history of prior successful VBAC (adjusted OR $0.23,95 \%$ CI $0.10-0.51$ ), and induction of labor (adjusted OR 2.53, 95\% CI 1.354.78). When we examined only women without a history of prior VBAC $(n=204)$ to determine significant covariates for the first failed VBAC, these included induction of labor (adjusted OR 2.46, 95\% CI 1.25-4.81) and chorioamnionitis (adjusted OR 4.43, 
Table 3. Comparison of Neonate Risk Factors and Neonatal Outcomes by Four Study Groups

\begin{tabular}{|c|c|c|c|c|c|}
\hline Neonate Characteristic & $\begin{array}{l}\text { Elective Cesarean } \\
\text { No Labor }(n=239)\end{array}$ & $\begin{array}{l}\text { Elective Cesarean } \\
\text { Labor }(n=104)\end{array}$ & $\begin{array}{l}\text { VBAC } \\
(n=244)\end{array}$ & $\begin{array}{l}\text { Failed VBAC } \\
\qquad(\mathrm{n}=\mathbf{8 5})\end{array}$ & $P^{*}$ \\
\hline \multicolumn{6}{|l|}{ Neonate disposition } \\
\hline Well baby nursery & $216(90.4)$ & $95(91.3)$ & $235(96.3)$ & $78(91.8)$ & .068 \\
\hline NICU & $23(9.6)$ & $9(8.7)$ & $9(3.7)$ & $7(8.2)$ & \\
\hline Gestational age (wk) & $39.1(37.0,41.0)$ & $39.1(37.3,41.0)$ & $39.5(37.4,41.3)$ & $40.0(37.4,41.4)$ & $<.001$ \\
\hline Male sex of neonate & $122(51.5)$ & $59(56.7)$ & $123(50.4)$ & $46(54.1)$ & .70 \\
\hline Birth weight $(\mathrm{kg})$ & $3.3(2.7,4.2)$ & $3.3(2.5,4.1)$ & $3.3(2.6,4.0)$ & $3.3(2.6,4.0)$ & .11 \\
\hline $\begin{array}{l}\text { Oxygen during delivery room } \\
\text { resuscitation }\end{array}$ & $96(40.2)$ & $46(44.2)$ & $46(18.9)$ & $30(35.3)$ & $<.001$ \\
\hline Most delivery room resuscitation needed & & & & & $<.001$ \\
\hline Routine & $142(59.4)$ & $58(55.8)$ & $196(80.3)$ & $55(64.7)$ & \\
\hline Blow-by oxygen & $66(27.6)$ & $34(32.7)$ & $27(11.1)$ & $16(18.8)$ & \\
\hline Mask CPAP & $22(9.2)$ & $10(9.6)$ & $13(5.3)$ & 0 & \\
\hline Bag/mask ventilation & $6(2.5)$ & $2(1.9)$ & $3(1.2)$ & $8(9.4)$ & \\
\hline Intubation & $2(0.8)$ & 0 & $3(1.2)$ & $5(5.9)$ & \\
\hline Admission for hypoglycemia & $9(3.8)$ & $3(2.9)$ & $2(0.8)$ & $1(1.2)$ & .14 \\
\hline Oxygen requirement in NICU & $14(5.9)$ & $6(5.8)$ & $3(1.2)$ & $5(5.9)$ & .04 \\
\hline Conventional ventilation & $1(0.42)$ & 0 & 0 & $2(2.4)$ & \\
\hline High frequency ventilation & 0 & 0 & 0 & 0 & \\
\hline Oxygen hood & $5(2.1)$ & $1(1.0)$ & 0 & $1(1.2)$ & \\
\hline Cannula & $6(2.5)$ & $1(1.0)$ & $1(1.2)$ & $1(1.2)$ & \\
\hline CPAP & $2(0.8)$ & $4(3.8)$ & $2(0.8)$ & $1(1.2)$ & \\
\hline
\end{tabular}

VBAC, vaginal birth after cesarean; NICU, neonatal intensive care unit; CPAP, continuous positive airway pressure.

Data are $\mathrm{n}(\%)$ and median $(5 \%, 95 \%)$.

* Continuous variables and categorical variables were compared with Kruskal Wallis and $\chi^{2}$ analyses, respectively.

95\% CI 1.54-12.77). Parity (adjusted OR 0.70, 95\% CI 0.53-0.92) and maternal age (adjusted OR 1.04, 95\% CI 1.00-1.11) were significant covariates predictive of failed VBAC.

Given the American College of Obstetrics and Gynecology recommendations that neonates 39 weeks or younger not be delivered by elective repeat cesarean delivery due to complications from iatrogenic prematurity and respiratory distress, we stratified neonatal outcomes by gestational age (Table 5). The 37-week group had the highest rates of oxygen need in the delivery room $(38.8 \%, P=.003)$, and

Table 4. Multivariable Logistic Regression Models Showing the Unadjusted and Adjusted Odds Ratio of the Categories of Delivery and Select Covariates for Admission to the Neonatal Intensive Care Unit

\begin{tabular}{lcccc}
\hline & $\begin{array}{c}\text { Unadjusted } \\
\text { OR }\end{array}$ & $\begin{array}{c}\text { Adjusted } \\
\text { OR }\end{array}$ & $\mathbf{9 5 \%} \mathrm{Cl}^{*}$ & $\boldsymbol{P}$ \\
\hline ERCD, no labor $^{\dagger}$ & 2.78 & 2.93 & $1.28-6.72$ & .011 \\
ERCD, with labor $^{\dagger}$ & 2.47 & 2.26 & $0.85-6.00$ & .100 \\
Failed VBAC $^{\dagger}$ & 2.34 & 1.91 & $0.66-5.58$ & .235 \\
Successful VBAC & 1.00 & 1.00 & & \\
\hline
\end{tabular}

OR, odds ratio; CI, confidence interval; ERCD, elective repeat cesarean delivery; VBAC, vaginal birth after cesarean.

* Values of $95 \%$ confidence interval and $P$ are for the adjusted odds ratio.

$\dagger$ Successful vaginal birth after cesarean as referent. admissions to the $\mathrm{NICU}(10.0 \%, P=.018)$, despite also having a greater number of amniocenteses for fetal lung maturity performed $(25.0 \%, P<.001)$ than the older neonates. A greater proportion of the 37 -week neonates required the support of continuous positive airway pressure $(3.8 \%)$ or supplemental oxygen $(2.6 \%)$ in the NICU than the older neonates, although this did not reach statistical significance.

The differences in length of hospital stay for mothers and neonates and hospital costs are shown in Table 6 and Table 7. For both mothers and neonates, successful VBAC was associated with the shortest length of hospital stay (median 4 days), compared with the other three groups (median 3 days). Overall, intended cesarean delivery was significantly more expensive than intended vaginal delivery for both mothers and their neonates. Both elective cesarean delivery with or without labor accrued higher costs compared with successful VBAC delivery. However, facility, physician, and total costs due to failed VBAC delivery significantly exceeded those of the other three groups $(P<.001)$ for both mothers and their neonates, except for neonate physician fees. Indeed, when determining the overall costs for the total birth (including delivery and NICU use) presented as median followed by $5 \%$ to $95 \%$, the failed VBAC group was the most expensive $(\$ 9,388,6,631$ to 16,275$)$, followed by elective cesarean delivery with labor 
Table 5. Comparison of Neonatal Outcomes by Completed Gestational Age in Weeks

\begin{tabular}{|c|c|c|c|c|c|}
\hline Neonate Characteristic & $37 \mathrm{wk}(\mathrm{n}=80)$ & $38 w k(n=116)$ & 39 wk $(n=289)$ & $\geq 40$ wk $(n=187)$ & $P$ \\
\hline \multicolumn{6}{|l|}{ Study group } \\
\hline ERCD, no labor & $29(36.2)$ & $32(27.6)$ & $148(51.2)$ & $30(16.0)$ & $<.001$ \\
\hline ERCD, with labor & $19(23.6)$ & $21(18.1)$ & $45(15.6)$ & $19(10.2)$ & \\
\hline VBAC & $24(30.0)$ & $48(41.4)$ & $79(27.3)$ & $93(50.0)$ & \\
\hline Failed VBAC & $8(10.0)$ & $15(12.9)$ & $17(5.9)$ & $45(24.1)$ & \\
\hline \multicolumn{6}{|l|}{ Neonate disposition } \\
\hline Well baby nursery & $68(85.0)$ & $112(96.6)$ & $270(93.1)$ & $175(93.6)$ & .018 \\
\hline NICU & $12(15.0)$ & $4(3.5)$ & $20(6.9)$ & $12(6.4)$ & \\
\hline Oxygen during delivery room resuscitation & $31(38.8)$ & $34(29.6)$ & $99(34.1)$ & $54(29.2)$ & .32 \\
\hline \multicolumn{6}{|l|}{ Most delivery room resuscitation needed } \\
\hline Routine & $49(61.3)$ & $81(69.8)$ & $191(65.9)$ & $131(70.1)$ & .003 \\
\hline Blow-by oxygen & $13(16.3)$ & $25(21.6)$ & $73(25.2)$ & $32(17.1)$ & \\
\hline Mask CPAP & $14(17.5)$ & $6(5.2)$ & $17(5.9)$ & $8(4.3)$ & \\
\hline $\mathrm{Bag} / \mathrm{mask}$ ventilation & $4(5.0)$ & 0 & $7(2.4)$ & $8(4.3)$ & \\
\hline Intubation & 0 & $3(2.6)$ & $2(0.7)$ & $5(2.7)$ & \\
\hline CPR, medications & 0 & 0 & 0 & $1(0.5)$ & \\
\hline Amniocentesis for FLM & $20(25.0)$ & $4(3.5)$ & $7(2.4)$ & $1(0.5)$ & $<.001$ \\
\hline Oxygen requirement in NICU & $5(6.3)$ & $3(2.6)$ & $12(4.1)$ & $8(4.3)$ & .66 \\
\hline Conventional ventilation & 0 & 0 & $2(0.7)$ & $1(0.5)$ & \\
\hline Oxygen hood & $1(1.3)$ & 0 & $3(1.0)$ & $3(1.6)$ & \\
\hline Cannula & $1(1.3)$ & 0 & $5(1.7)$ & $3(1.6)$ & \\
\hline CPAP & $3(3.8)$ & $3(2.6)$ & $2(0.7)$ & $1(0.5)$ & \\
\hline
\end{tabular}

ERCD, elective repeat cesarean delivery; VBAC, vaginal birth after cesarean; NICU, neonatal intensive care unit; CPAP, continuous positive airway pressure; FLM, fetal lung maturity.

$(\$ 8,331,5,995$ to 11,310$)$, elective cesarean delivery without labor $(\$ 8,239,6,212$ to 13,968$)$, with successful VBAC being the least expensive $(\$ 5,853,3,793$ to 10,333). Despite the greatest costs being seen in the failed VBAC group, the intended elective repeat cesarean delivery group still accrued significantly higher facility, physician, and total costs than the intended VBAC group.

\section{DISCUSSION}

Our study demonstrates that neonates born by intended elective repeat cesarean delivery, with or without labor, have increased rates of admission to the NICU compared with the intended VBAC group. First, we found that those neonates born by intended cesarean delivery were more prone to NICU admission for hypoglycemia than neonates in the intended VBAC group. In addition, our results show that more neonates born by intended elective repeat cesarean delivery required higher rates of oxygen supplementation and ventilatory support in the NICU, compared with neonates born in the intended VBAC group. These findings are consistent with multiple studies documenting respiratory morbidity in neonates born after elective repeat cesarean delivery, particularly with an increase in respiratory distress syndrome, transient tachypnea of the newborn, persistent pulmonary hypertension, and need for supplemental oxygen. ${ }^{3,11}$ Respiratory morbidity as a result of failure to clear fetal lung fluid is common and can be challenging for neonates delivered by elective repeat cesarean delivery without being exposed to labor. We hypothesize that the catecholamine surge that occurs during labor likely plays an important role in both clearance of fetal lung fluid and glycemic control after birth. While the common perception is that condi-

Table 6. Comparison of Length and Cost in Dollars of Hospital Stay Between the Intended Cesarean and Intended Vaginal Birth After Cesarean Groups

\begin{tabular}{lccc}
\hline Type of Costs* & Intended Cesarean Group $(\mathrm{n}=343)$ & Intended VBAC Group $(\mathbf{n}=329)^{\boldsymbol{P}^{+}}$ \\
\hline Mother total cost & $6,044(4,391,8,677)$ & $4,835(2,862,9,033)$ & $<.001$ \\
Neonate total cost & $2,099(1,414,4,649)$ & $1,526(820,3,569)$ & $<.001$ \\
Total costs for birth & $8,268(6,179,13,812)$ & $6,647(3,899,13,286)$ & $<.001$ \\
\hline
\end{tabular}

VBAC, vaginal birth after cesarean.

Data are median $(5 \%, 95 \%)$.

* Adjusted for inflation to 2008 U.S. dollars according to the Consumer Price Index ${ }^{10}$ and discounting by $3 \%$ yearly. ${ }^{9}$

$\dagger P$ values were calculated by Kruskal-Wallis test. 
Table 7. Comparison of Length and Cost in Dollars of Hospital Stay Among Four Study Groups

\begin{tabular}{|c|c|c|c|c|c|}
\hline Type of Costs* & $\begin{array}{c}\text { Elective Cesarean No } \\
\text { Labor }(n=239)\end{array}$ & $\begin{array}{l}\text { Elective Cesarean } \\
\text { Labor }(n=104)\end{array}$ & VBAC $(n=244)$ & $\begin{array}{l}\text { Failed VBAC } \\
\quad(n=85)\end{array}$ & $P^{+}$ \\
\hline Mother length of stay & $4.0(3,5)$ & $4.0(3,5)$ & $3.0(2,4)$ & $4.0(3,6)$ & $<.001$ \\
\hline Mother facility cost & $4,696(3,020,6,835)$ & $4,859(3,450,6,135)$ & $3,344(2,116,5,680)^{\S}$ & $5,801(3,927,9,129)^{\|}$ & $<.001$ \\
\hline Mother physician cost & $1,353(678,2,184)$ & $1,253(675,2,114)^{\uparrow}$ & $922(481,1,624)^{\S}$ & $1,495(974,2,259)^{\|}$ & $<.001$ \\
\hline Mother total cost & $6,030(4,543,8,572)$ & $6,073(4,319,8,745)^{\uparrow}$ & $4,345(2,761,7,374)^{\S}$ & $7,292(5,295,11,162)^{\|}$ & $<.001$ \\
\hline Neonate length of stay & $4.0(3,5)$ & $4.0(3,5)$ & $3.0(2,4)$ & $4.0(3,5)$ & $<.001$ \\
\hline Neonate facility cost & $2,020(1,299,4,567)$ & $2,018(1,318,3,925)$ & $1,302(719,2,711)$ & $2,033(1,294,5,289)$ & $<.001$ \\
\hline Neonate physician cost & $92(42,372)^{\#}$ & $82(41,853)^{* *}$ & $80(42,301)^{\dagger \dagger}$ & $82(41,268)^{\ddagger}$ & .466 \\
\hline Neonate total cost & $2,094(1,400,4,742)^{\#}$ & $2,103(1,414,4,007)^{* *}$ & $1,453(797,3,067)^{\dagger \dagger}$ & $2,129(1,336,5,470)$ 市 & $<.001$ \\
\hline Total costs for birth & $8,239(6,212,13,968)^{\#}$ & $8,331(5,995,11,310)^{* *}$ & $5,853(3,793,10,333)^{\dagger \dagger}$ & $9,338(6,631,16,275)^{\text {市 }}$ & $<.001$ \\
\hline
\end{tabular}

VBAC, vaginal birth after cesarean.

Data are median $(5 \%, 95 \%)$.

* Adjusted for inflation to 2008 U.S. dollars according to the Consumer Price Index ${ }^{10}$ and discounting by $3 \%$ yearly. ${ }^{9}$

$\dagger P$ values were calculated by Kruskal Wallis Test.

$¥$ Length of stay is presented as median, and 5\% and 95\% in parentheses.

$\S$ Final calculations based on the 241 records that had data for all categories.

"Final calculations based on the 84 records that had data for all categories.

" Final calculations based on the 102 records that had data for all categories.

\# Final calculations based on the 193 records that had data for all categories.

** Final calculations based on the 88 records that had data for all categories.

$\dagger$ Final calculations based on the 188 records that had data for all categories.

$¥$ Final calculations based on the 76 records that had data for all categories.

tions such as transient tachypnea of the newborn are benign, self-limiting illnesses, several studies indicate that neonates with such conditions can progress to severe respiratory failure, leading to the need for extracorporeal membrane oxygenation or death. ${ }^{12}$

The differences seen between the intended elective repeat cesarean delivery and VBAC groups take on greater significance when one notes that the intended VBAC group includes neonates born after failed VBAC delivery, who required the greatest measures of resuscitation due to fetal distress, characterized by nonreassuring fetal heart tones and meconium-stained amniotic fluid. At the other extreme, neonates born after successful VBAC had the lowest rates of admission to the NICU, shortest hospital stay, and the lowest incidence of ongoing respiratory support. Further investigation of the ability to select those women with the greatest potential for success with a trial of labor will enable a woman and her neonate to enjoy the best possible outcomes and avoid the worst outcomes. A nomogram recently published for prediction of successful VBAC includes maternal factors, including age, BMI, maternal race, any prior vaginal delivery, any prior $\mathrm{VBAC}$, and recurring indication for cesarean delivery. ${ }^{13}$ Our multivariable analyses suggest that risk factors during delivery, including chorioamnionitis and induction of labor, are associated with VBAC failure, and that older maternal age and lower parity were also predictive of VBAC failure.

Our results regarding the differential costs of delivery and NICU use among the four study groups lends support to the notion of improving the selection of women to successfully undergo a trial of labor. Indeed, the costs for the total birth (including delivery fees for the mother and NICU use fees for the neonate) for neonates born by successful VBAC delivery were the lowest of all four groups. Although our study adds to the currently sparse information about costs of elective repeat cesarean delivery compared with trial of labor, we were not able to perform further postpartum follow-up of the mothers to determine the additional costs accrued by postsurgical complications. Larger multicenter databases might allow for better ascertainment of the incidence of rarer maternal and neonatal outcomes and their effects on cost. Moreover, we did not have the data to allow us to estimate costs other than those for hospital care. For instance, we were unable to measure patient and family costs such as the cost of parking or meals, or societal costs such as productivity loss, which might differ among the treatment conditions. ${ }^{8}$

Clearly, rates of respiratory morbidity were lower after onset of labor and with increasing gestational age. ${ }^{11,14,15}$ Indeed, several studies note that the incidence of respiratory distress is significantly greater in those neonates less than 39 weeks gestation ${ }^{11,14-16}$; therefore, accurate dating of the mother's pregnancy before a decision regarding elective repeat cesarean delivery is necessary. Our findings suggest strict adherence to the American College of Obstetrics and Gynecology guidelines that neonates less than 39 weeks gestation not be delivered by elective repeat 
cesarean delivery. Interestingly, although the overall rates of amniocenteses were low, the greatest number of amniocenteses for fetal lung maturity were performed in those neonates who had an elective repeat cesarean delivery without labor. Still, these neonates continued to have high rates of respiratory morbidity and NICU admission, indicating that surfactant deficiency may not be the sole cause of respiratory distress seen after elective cesarean delivery. ${ }^{3}$ In addition, although fetal lung maturity is thought to be a surrogate for physiologic maturation, several recent studies have called for a shift in thinking of "term" neonates as being 37 weeks and greater, and that perhaps those neonates between $370 / 7$ weeks and 38 $6 / 7$ weeks be called "early term" to identify their immaturity relative to those neonates at 39 weeks or greater. ${ }^{17}$

Women would not have been considered for this study if they had not undergone a primary cesarean delivery in the first place. Given the increasing rates of primary cesarean delivery and the concomitant decrease in VBACs, once a woman has had a primary cesarean delivery, we must consider the risks that this places on her subsequent deliveries and subsequent neonates. Indeed, this argues for greater selectivity in performing a cesarean delivery in the first place, and certainly a greater need for counseling before a primary elective cesarean delivery. As investigators continue to search for ways to make cesarean delivery safer, we may be better served by exploring other means for reducing overall cesarean delivery rates and recognizing our own preoccupation with the individual that will be our patient, whether it be mother or neonate.

\section{REFERENCES}

1. Hamilton BE, Martin JA, Ventura SJ. Births: Preliminary Data for 2006. National Vital Statistics Reports. December 5, 2007. Volume 56, Number 7. Available from www.cdc.gov/ nchs/data/nvsr/nvsr56/nvsr56_07.pdf. Retrieved November 8, 2008.

2. MacDorman MF, Menacker F, Declercq E. Cesarean birth in the United States: epidemiology, trends, and outcomes. Clin Perinatol 2008;35:293-307.
3. Ramachandrappa A, Jain L. Elective cesarean section: its impact on neonatal respiratory outcome. Clin Perinatol 2008; 35:373-93.

4. Spong CY, Landon MB, Gilbert S, Rouse DJ, Leveno KJ, Varner MW, et al. Risk of uterine rupture and adverse perinatal outcome at term after cesarean delivery. Obstet Gynecol 2007;110:801-7.

5. Smith GC, Pell JP, Cameron AD, Dobbie R. Risk of perinatal death associated with labor after previous cesarean delivery in uncomplicated term pregnancies. JAMA 2002;287:2684-90.

6. Fetal maturity assessment prior to elective repeat cesarean delivery. ACOG Committee Opinion: Committee on Obstetrics: Maternal and Fetal Medicine. Number 98-September 1991 (replaces No. 77, January 1990). J Gynaecol Obstet 1992; 38: 327.

7. National Institutes of Health. NIH Consensus Development Program. NIH State-of-the-Science Conference: cesarean delivery on maternal request. March 27-29, 2006 Available at: http://consensus.nih.gov/2006/2006CesareanSOS027main.htm. Retrieved March 26, 2009.

8. Zupancic JA. The economics of elective cesarean section. Clin Perinatol 2008;35:591-9.

9. Gold MR, Siegel JE, Russell LB, Weinstein MC. Cost-effectiveness in health and medicine. New York (NY): Oxford University Press; 1996

10. U.S. Bureau of Labor Statistics. Division of Consumer Prices and Price Indexes. Consumer price index. Available at: http:// www.bls.gov/cpi/home.htm. Retrieved November 6, 2008.

11. Hansen AK, Wisborg K, Uldbjerg N, Henriksen TB. Risk of respiratory morbidity in term infants delivered by elective cesarean section: cohort study. BMJ 2008;336:85-7.

12. Keszler M, Carbone MT, Cox C, Schumacher RE. Severe respiratory failure after elective repeat cesarean delivery: a potentially preventable condition leading to extracorporeal membrane oxygenation. Pediatrics 1992;89:670-2.

13. Grobman WA, Lai Y, Landon MB, Spong CY, Leveno KJ, Rouse DJ, et al. Development of a nomogram for prediction of vaginal birth after cesarean delivery. Obstet Gynecol 2007; 109:806-12.

14. Yee W, Amin H, Wood S. Elective cesarean delivery, neonatal intensive care unit admission, and neonatal respiratory distress. Obstet Gynecol 2008;111:823-8.

15. Yoder BA, Gordon MC, Barth WH Jr. Late-preterm birth: does the changing obstetric paradigm alter the epidemiology of respiratory complications? Obstet Gynecol 2008;111:814-22.

16. Tita AT, Landon MB, Spong CY, Lai Y, Leveno KJ, Varner MW, et al. Timing of elective repeat cesarean delivery at term and neonatal outcomes. N Engl J Med 2009;360:111-20.

17. Engle WA, Kominiarek MA. Late preterm infants, early term infants, and timing of elective deliveries. Clin Perinatol 2008;35:325-41. 